\title{
Mesh Variational Autoencoders with Edge Contraction Pooling
}

\author{
Yu-Jie Yuan ${ }^{\mathrm{a}, \mathrm{b}}$, Yu-Kun Lai ${ }^{\mathrm{c}}$, Jie Yang ${ }^{\mathrm{a}, \mathrm{b}}$, Qi Duan ${ }^{\mathrm{d}}$, Hongbo Fu ${ }^{\mathrm{e}}$ and Lin Gao ${ }^{\mathrm{a}, \mathrm{b}, *}$ \\ ${ }^{a}$ Beijing Key Laboratory of Mobile Computing and Pervasive Device, Institute of Computing Technology, CAS \\ ${ }^{\mathrm{b}}$ University of Chinese Academy of Sciences \\ ${ }^{\mathrm{c}}$ School of Computer Science and Informatics, Cardiff University, UK \\ ${ }^{\mathrm{d}}$ SenseTime Research \\ ${ }^{\mathrm{e}}$ City University of Hong Kong
}

\begin{abstract}
$3 D$ shape analysis is an important research topic in computer vision and graphics. While existing methods have generalized image-based deep learning to meshes using graph-based convolutions, the lack of an effective pooling operation restricts the learning capability of their networks. In this paper, we propose a novel pooling operation for mesh datasets with the same connectivity but different geometry, by building a mesh hierarchy using mesh simplification. For this purpose, we develop a modified mesh simplification method to avoid generating highly irregularly sized triangles. Our pooling operation effectively encodes the correspondence between coarser and finer meshes in the hierarchy. We then present a variational auto-encoder (VAE) structure with the edge contraction pooling and graphbased convolutions, to explore probability latent spaces of $3 D$ surfaces and perform $3 D$ shape generation. Our network requires far fewer parameters than the original mesh VAE and thus can handle denser models thanks to our new pooling operation and convolutional kernels. Our evaluation also shows that our method has better generalization ability and is more reliable in various applications, including shape generation and shape interpolation.
\end{abstract}

\section{Introduction}

In recent years, 3D shape datasets have been increasingly available on the Internet. Consequently, data-driven 3D shape analysis has been an active research topic in computer vision and graphics. Apart from traditional datadriven works such as [7], recent works attempted to generalize deep neural networks from images to 3D shapes such as [30, 31, 18] for triangular meshes, [24] for point clouds, $[35,21]$ for voxel data, etc. In this paper, we concentrate on

*Corresponding Author: gaolin@ict.ac.cn (Lin Gao) deep neural networks for triangular meshes. Unlike images, 3D meshes have complex and irregular connectivity. Most existing works tend to keep mesh connectivity unchanged from layer to layer, thus losing the capability of increased receptive fields when pooling operations are applied.

As a generative network, the Variational Auto-Encoder (VAE) [16] has been widely used in various kinds of generation tasks, including generation, interpolation and exploration on triangular meshes [31]. The original MeshVAE [31] uses a fully connected network that requires a huge number of parameters and its generalization ability is often weak. Although the fully connected layers allow changes of mesh connectivity between layers, due to irregular changes, such approaches cannot be directly generalized to convolutional layers. Some works $[18,9]$ adopt convolutional layers in the VAE structure. However, such convolution operations cannot change the connectivity of the mesh. The work [26] introduces sampling operations in CNNs on meshes, but their sampling strategy does not aggregate all the local neighborhood information when reducing the number of vertices. Therefore, in order to deal with denser models and enhance the generalization ability of the network, it is necessary to design a pooling operation for meshes similar to the pooling for images to reduce the number of network parameters. Moreover, it is desired that the defined pooling can support further convolutions and allow recovery through a corresponding de-pooling operation.

In this paper we propose a VAE architecture with newly defined pooling operations. Our method uses mesh simplification to form a mesh hierarchy with different levels of details, and achieves effective pooling by keeping track of the mapping between coarser and finer meshes. To avoid generating highly irregular triangles during mesh simplification, we introduce a modified mesh simplification approach based on [11]. The input to our network is a vertexbased deformation feature representation [8], which unlike 3D coordinates, encodes deformations using deformation 
gradients defined on vertices. Our framework uses a collection of 3D shapes with the same connectivity to train the network. Such meshes can be easily obtained through consistent remeshing. Also, we adopt graph convolutions [6] in our network. In all, our network follows a VAE architecture where pooling operations and graph convolutions are applied. As we will show later, our network not only has better generalization capabilities but also can handle much higher resolution meshes, benefiting various applications, such as shape generation and interpolation.

\section{Related Work}

Deep Learning for 3D Shapes. Deep learning on 3D shapes has received increasing attention. Boscaini et al. [2, 3] generalize CNNs from the Euclidean domain to the nonEuclidean domain, which is useful for 3D shape analysis such as establishing correspondences. Bronstein et al. [5] give an overview of utilizing CNNs on non-Euclidean domains, including graphs and meshes. Masci et al. [20] propose the first mesh convolutional operations by applying filters to local patches represented in geodesic polar coordinates. Maron et al. [19] parameterize a surface to a planar flat-torus to define a natural convolution operator for CNNs on surfaces. Wang et al. [33, 34] propose octree-based convolutions for 3D shape analysis. Unlike local patches, planar flat-tori, or octrees, our work performs convolutional operations using vertex features [8] as input.

To analyze meshes with the same connectivity but different geometry, the work [31] first introduced the VAE architecture to 3D mesh data, and demonstrates its usefulness using various applications. Tan et al. [30] use a convolutional auto-encoder to extract localized deformation components from mesh datasets with large-scale deformations. Gao et al. [9] propose a network that combines convolutional mesh VAEs with CycleGAN [37] for automatic unpaired deformation transfer. Their follow-up work [10] further proposes a two-level VAE for generating 3D shapes of man-made objects with fine geometry details and complex structures. The works [30,9] apply convolutional operations to meshes in the spatial domain, while the works of $[6,13]$ extend CNNs to irregular graphs by construction in the spectral domain, and show superior performance when compared with spatial convolutions. Following [6, 36], our work also performs convolutional operations in the spectral domain.

While pooling operations have been widely used in deep networks for image processing, existing mesh-based VAE methods either do not support pooling [31,9], or use a simple sampling process [26], which is not able to aggregate all the local neighborhood information. In fact, the sampling approach in [26], although also based on a simplification algorithm, directly drops vertices, and uses the barycentric coordinates in triangles of the coarse mesh to recover the lost vertices by interpolation. In contrast, our pooling op- erations can aggregate local information by recording the simplification procedure, and support direct reversal of the pooling operation to effectively achieve de-pooling. More recently, Hanocka et al. [12] proposed MeshCNN, containing a dynamic mesh pooling operation, which conducts mesh simplification according to specific tasks. On the contrary, we define our pooling operation based on a static mesh simplification algorithm, aiming for generating high quality mesh models. The static algorithm ensures consistent hierarchies, so better preserves geometric details and is more robust.

Uniform Sampling or Pooling Methods. Taking point clouds as input, PointNet++ [25] proposes a uniform sampling method for point cloud based neural networks. Using the same idea, TextureNet [14] also conducts uniform sampling on the vertices of a mesh. This kind of sampling method destroys the connection between vertices, turning mesh data into a point cloud, which cannot support further graph convolutions. In contrast, simplification methods can build mesh hierarchies, so can help us perform mesh pooling operations. However, most simplification methods, such as [11], are shape-preserving, but vertices on the simplified meshes can be highly non-uniform. Remeshing operations such as [4], on the other hand, can build uniform simplified meshes, but lose the correspondence between meshes in the hierarchy. We propose a modified mesh simplification method based on the classic method [11] to simplify meshes more uniformly and record the correspondences between the coarse and dense meshes for newly defined mesh pooling and de-pooling operations.

\section{Our Framework}

In this section we introduce the basic operations and network architecture used in our framework.

\subsection{Mesh Simplification}

We use mesh simplification to help build reliable pooling operations. For this purpose, mesh simplification not only creates a mesh hierarchy with different levels of details, but also ensures the correspondences between coarser and finer meshes. Our simplification process is based on the classical method [11], which performs repeated edge contraction in an order based on a metric measuring shape changes. However, the original approach cannot guarantee that the simplified mesh contains evenly distributed triangles. To achieve more effective pooling, each vertex in the coarser mesh should correspond to a similarly sized region.

Our observation is that the edge length is an important indicator for this process. To avoid contracting long edges, we incorporate the edge length as one of the criteria to order pairs of points to be simplified. The original work defines the error at vertex $\mathbf{v}=\left[v_{x}, v_{y}, v_{z}, 1\right]^{\mathrm{T}}$ to be a quadratic form $\mathbf{v}^{\mathrm{T}} \mathbf{Q} \mathbf{v}$, where $\mathbf{Q}$ is the sum of the fundamental error 


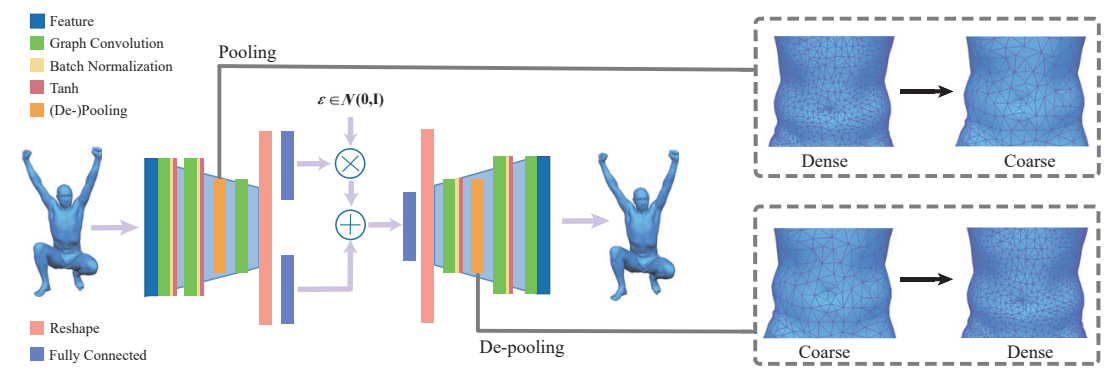

Figure 1. Our network architecture. $\epsilon$ is a random variable with a Gaussian distribution with 0 mean and unit variance.

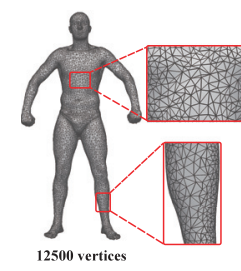

(a) Original Mesh

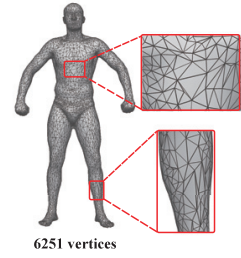

(b) Garland and Heckbert 1997

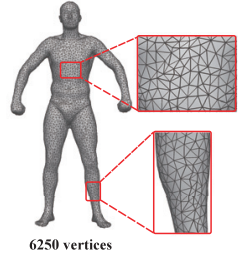

(c) Our Method
Figure 2. Comparison of the mesh simplification algorithm [11] and our modified version. (a) the original mesh with 12,500 vertices, (b) a result of [11] with 6,251 vertices, and (c) our result with 6,250 vertices.

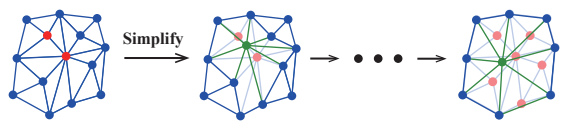

Figure 3. We use a simplification algorithm to introduce our pooling operation on meshes. The red vertices are simplified to the green vertex by edge contraction and the features of the red vertices are averaged to give the feature of the green vertex.

quadrics introduced in [11]. For a given edge contraction $\left(\mathbf{v}_{1}, \mathbf{v}_{2}\right) \rightarrow \overline{\mathbf{v}}$, they simply choose to use $\overline{\mathbf{Q}}=\mathbf{Q}_{1}+\mathbf{Q}_{2}$ to be the new matrix which approximates the error at $\overline{\mathbf{v}}$. So the error at $\overline{\mathbf{v}}$ will be $\overline{\mathbf{v}}^{\mathrm{T}} \overline{\mathbf{Q}} \overline{\mathbf{v}}$. We propose to add the new edge length to the original simplification error metric. Specifically, given an edge $\left(\mathbf{v}_{i}, \mathbf{v}_{j}\right)$ to be contracted to a new vertex $\overline{\mathbf{v}}_{k}$, the total error is defined as:

$$
\begin{aligned}
E & =\overline{\mathbf{v}}_{k}^{\mathrm{T}} \overline{\mathbf{Q}}_{k} \overline{\mathbf{v}}_{k} \\
& +\gamma \max \left\{L_{k m}, L_{k n} \mid m \in \mathcal{N}_{i}, n \in \mathcal{N}_{j}, m \neq j, n \neq i\right\},
\end{aligned}
$$

where $L_{k m}$ (resp. $L_{k n}$ ) is the new edge length between vertex $k$ and vertex $m$ (resp. vertex $n)$. $\mathcal{N}_{i}\left(\right.$ resp. $\left.\mathcal{N}_{j}\right)$ is the set of neighboring vertices of vertex $i$ (resp. vertex $j$ ), and $\lambda$ is a weight. Note that we only penalize the maximum edge length around newly created vertices $\overline{\mathbf{v}}_{k}$ to effectively avoid triangles with too long edges. In our experiments, we contract half of the vertices between adjacent levels of details to support effective pooling. A representative simplification example is shown in Fig. 2, which clearly shows the effect of our modified simplification algorithm. The advantage of our modified simplification algorithm over the original one on pooling and thus shape reconstruction will be discussed in Section 4.1.

\subsection{Pooling and De-pooling}

Mesh simplification is achieved by repeated edge contraction, i.e., contracting two adjacent vertices to a new vertex. We exploit this process to define our pooling operation, in a way similar to image-based pooling. We use average pooling for our framework (and alternative pooling operations can be similarly defined). As illustrated in Fig. 3, following an edge contraction step, we define the feature of a new vertex as the average feature of the contracted vertices. This ensures that the pooling operation effectively operates at relevant simplified regions. This process has some advantages: It preserves a correct topology to support multiple levels of convolutions/pooling, and makes the receptive field well defined.

Since our network has a decoder structure, we also need to properly define a de-pooling operation. We similarly take advantage of simplification relationships, and define de-pooling as the inverse operation: the features of the vertices on the simplified mesh are equally assigned to the corresponding contracted vertices on the dense mesh.

\subsection{Graph Convolution}

To form a complete neural network architecture, we adopt the spectral graph convolutions introduced in [6]. Let $\mathbf{x}$ be the input and $\mathbf{y}$ be the output of a convolution operation. $\mathbf{x}$ and $\mathbf{y}$ are matrices where each row corresponds to a vertex and each column corresponds to a feature dimension. Let $\mathbf{L}$ denote the normalized graph Laplacian. The spectral graph convolution used in our network is then defined as

$$
\mathbf{y}=g_{\theta}(\mathbf{L}) \mathbf{x}=\sum_{h=0}^{H-1} \theta_{h} \mathbf{T}_{h}(\tilde{\mathbf{L}}) \mathbf{x},
$$

where $\tilde{\mathbf{L}}=2 \mathbf{L} / \lambda_{\max }-\mathbf{I}, \lambda_{\max }$ is the largest eigenvalue, $\theta \in \mathbb{R}^{H}$ is polynomial coefficients, and $\mathbf{T}_{h}(\tilde{\mathbf{L}}) \in \mathbb{R}_{\tilde{\tilde{\mathbf{L}}}}^{V \times V}$ is the Chebyshev polynomial of order $h$ evaluated at $\tilde{\mathbf{L}}$.

\subsection{Network Structure}

As illustrated in Fig. 1, our network is built on our average pooling operation and convolutional operation, with a variational auto-encoder structure. The input to the encoder is the preprocessed ACAP (As-Consistent-As- 
Possible) features [8] with each dimension linearly scaled to $[-0.95,0.95]$ to allow using $\tanh$ as activation function, which are shaped as $X \in \mathbb{R}^{V \times 9}$, where $V$ is the number of vertices and 9 is the dimension of the deformation representation. The representation effectively encodes local deformations and copes well with large rotations.

Unlike the original mesh VAE [31], which uses fully connected layers, the encoder of our network consists of two graph convolutional layers and one pooling layer followed by another graph convolutional layer. The output of the last convolutional layer is mapped to a mean vector and a deviation vector by two different fully-connected layers. The mean vector does not have an activation function, and the deviation vector uses sigmoid as the activation function.

The decoder mirrors the encoder steps. However, we use different convolutional weights from the corresponding layers in the encoder, with all layers using the tanh output activation function. Corresponding to the pooling operation, the de-pooling operation as described in Section 3.2 maps features in a coarser mesh to a finer mesh. The output of the whole network is $\hat{X} \in \mathbb{R}^{V \times 9}$, which has the identical dimension as the input, and can be rescaled back to the deformation representation and used for reconstructing the deformed shape.

In order to train our VAE network, we use the mean squared error (MSE) as the reconstruction loss. Combined with the KL-divergence [17], the total loss function for the model is defined as

$$
L=\frac{1}{2 M} \sum_{i=1}^{M}\left\|X^{i}-\hat{X}^{i}\right\|_{F}^{2}+\alpha D_{K L}(q(z \mid X) \| p(z)),
$$

where $X^{i}$ and $\hat{X}^{i}$ represent the preprocessed features of the $i^{\text {th }}$ model and the output of the network. $\|\cdot\|_{F}$ is the Frobenius norm of matrix, $M$ is the number of shapes in the dataset, $\alpha$ is a parameter to adjust the priority between the reconstruction loss and KL-divergence. $z$ is the latent vector, $p(z)$ is the prior probability, $q(z \mid X)$ is the posterior probability, and $D_{K L}$ is the KL-divergence.

\subsection{Conditional VAE}

When the VAE is used for shape generation, it is often preferred to allow the selection of shape types to be generated, especially for datasets containing shapes from different categories (such as men and women, thin and fat, see [23] for more examples). To achieve this, we refer to [28] and add labels to the input and the latent vectors to extend our framework. In this case, our loss function is changed to

$$
L_{c}=\frac{1}{2 M} \sum_{i=1}^{M}\left\|X_{c}^{i}-\hat{X}^{i}\right\|_{F}^{2}+\alpha D_{K L}(q(z \mid X, c) \| p(z \mid c)),
$$

where $\hat{X}$ is the output of the conditional VAE, and $p(z \mid c)$ and $q(z \mid X, c)$ are conditional prior and posterior probabili- ties, respectively.

\subsection{Implementation Details}

In our experiments, we contract half of the vertices with $\gamma=0.001$ in Eq. 1 and set the hyper-parameter $H=3$ in graph convolutions, $\alpha=0.3$ in the total loss function. The latent space dimension is 128 for all our experiments. We also use $L_{2}$ regularization on the network weights to avoid over-fitting. We use Adam optimizer [15] with the learning rate set to 0.001 .

\section{Experiments}

\subsection{Framework Evaluation}

To compare different network structures and settings, we use several shape deformation datasets, including SCAPE dataset [1], Swing dataset [32], Face dataset [22], Horse and Camel dataset [29], Fat (ID:50002) from the MPI DYNA dataset [23], and Hand dataset. For each dataset, it is randomly split into halves for training and testing. We test the capability of the network to generate unseen shapes, and report the average RMS (root mean squared) errors.

Effect of Pooling. In Table 1 (Columns 3 and 8) we compare the RMS errors of reconstructing unseen shapes with and without pooling. The RMS error is lower by an average of $6.92 \%$ with pooling. The results show the benefit of our pooling and de-pooling operations.

Ablation Study. We compare spectral graph convolutions with alternative spatial convolutions, both with the network as shown in Fig. 1. The comparison results are shown in Table 1 (Columns 2 and 3). One can easily find that spectral graph convolutions give better results. Moreover, to demonstrate the benefit of our simplification-based pooling operation, we compare our pooling with the original simplification algorithm [11] for pooling, a representative uniform remeshing method [4] for pooling, the existing graph pooling method [27], and the mesh sampling operation [26]. Our method aims for a uniform, but also shape-preserving simplification, which leads to better generalization ability. The results are shown in Table 1.

Comparison with State-of-the-Art. In Table 2, we compare our method with the state-of-the-art mesh-based autoencoder architectures [9, 26, 31] in terms of RMS errors of reconstructing unseen shapes. We also compare with MeshCNN [12] in Table 3. We modify the segmentation network of MeshCNN for the encoding-decoding task. Thanks to spectral graph convolutions and our pooling, our method consistently reduces the reconstruction errors of unseen data, showing superior generalizability. We further show qualitative reconstruction comparison with [9] and [26] in Fig. 4. It shows that our method leads to more accurate reconstruction results than $[9,26]$. We also perform an experiment to illustrate that our network requires far fewer 


\begin{tabular}{|c|c|c|c|c|c|c|c|}
\hline Dataset & $\begin{array}{c}\text { Only } \\
\text { Spatial Conv. }\end{array}$ & $\begin{array}{c}\text { Only } \\
\text { Spectral Conv. }\end{array}$ & $\begin{array}{c}\text { Pooling } \\
\text { with [1] }\end{array}$ & $\begin{array}{c}\text { Uniform } \\
\text { Remeshing }\end{array}$ & $\begin{array}{c}\text { Graph } \\
\text { Pooling }\end{array}$ & $\begin{array}{c}\text { Mesh } \\
\text { Sampling }\end{array}$ & $\begin{array}{c}\text { Our } \\
\text { Method }\end{array}$ \\
\hline SCAPE & 0.1086 & 0.0825 & 0.0898 & 0.0813 & 0.0824 & 0.0831 & $\mathbf{0 . 0 7 6 3}$ \\
\hline Swing & 0.0359 & 0.0282 & 0.0284 & 0.0281 & 0.0292 & 0.0298 & $\mathbf{0 . 0 2 6 8}$ \\
\hline Fat & 0.0362 & 0.0267 & 0.0285 & 0.0305 & 0.0253 & 0.0289 & $\mathbf{0 . 0 2 4 9}$ \\
\hline Hand & 0.0300 & 0.0284 & 0.0271 & 0.0280 & 0.0306 & 0.0278 & $\mathbf{0 . 0 2 6 0}$ \\
\hline
\end{tabular}

Table 1. Comparison of RMS (root mean square) reconstruction errors for unseen data using our network with pooling ('Our Method'), without pooling ('Only Spectral Conv.'), without pooling and with an alternative spatial convolution operator ('Only Spatial Conv.'), with original simplification [11]-based pooling, with uniform remeshing [4], with graph pooling [27] and with mesh sampling [26].

\begin{tabular}{|c|c|c|c|c|c|}
\hline Dataset & \#. Vertices & $\begin{array}{c}\text { Tan } \\
2018\end{array}$ & $\begin{array}{c}\text { Gao } \\
2018\end{array}$ & $\begin{array}{c}\text { Ranjan } \\
2018\end{array}$ & Ours \\
\hline SCAPE & 12500 & - & 0.1086 & 0.1095 & $\mathbf{0 . 0 7 6 3}$ \\
\hline Swing & 9971 & - & 0.0359 & 0.0557 & $\mathbf{0 . 0 2 6 8}$ \\
\hline Fat & 6890 & 0.0308 & 0.0362 & 0.0324 & $\mathbf{0 . 0 2 4 9}$ \\
\hline Hand & 3573 & 0.0362 & 0.0300 & 0.0632 & $\mathbf{0 . 0 2 6 0}$ \\
\hline Face & 11849 & - & 1.0619 & 1.1479 & $\mathbf{0 . 7 2 5 7}$ \\
\hline Horse & 8431 & - & 0.0128 & 0.0510 & $\mathbf{0 . 0 1 1 9}$ \\
\hline Camel & 11063 & - & 0.0134 & 0.0265 & $\mathbf{0 . 0 1 1 5}$ \\
\hline
\end{tabular}

Table 2. Comparison of RMS reconstruction errors for unseen data using different auto-encoder frameworks proposed by Tan et al. [31], Gao et al. [9], and Ranjan et al. [26]. '-' means the corresponding method runs out of memory (largely due to the use of fully connected networks).

\begin{tabular}{|c|c|c|c|c|}
\hline Dataset & \multicolumn{2}{|c|}{ SCAPE } & \multicolumn{2}{c|}{ Swing } \\
\hline Method & MeshCNN & Ours & MeshCNN & Ours \\
\hline dihedral angle & 0.0690 & $\mathbf{0 . 0 0 0 6}$ & 0.0506 & $\mathbf{0 . 0 0 0 3}$ \\
\hline inner angle 1 & 0.3245 & $\mathbf{0 . 0 6 1 4}$ & 0.3713 & $\mathbf{0 . 0 4 2 1}$ \\
\hline inner angle 2 & 0.3100 & $\mathbf{0 . 0 5 2 9}$ & 0.2964 & $\mathbf{0 . 0 4 0 2}$ \\
\hline edge-length ratio 1 & 0.3806 & $\mathbf{0 . 0 6 6 1}$ & 0.3645 & $\mathbf{0 . 0 5 3 7}$ \\
\hline edge-length ratio 2 & 0.3668 & $\mathbf{0 . 0 6 4 9}$ & 0.3523 & $\mathbf{0 . 0 4 7 5}$ \\
\hline
\end{tabular}

Table 3. Comparison of MAE (mean absolute error) reconstruction errors with MeshCNN [12]. We use MAE of the five edge features, which are the inputs of MeshCNN, as the metric.

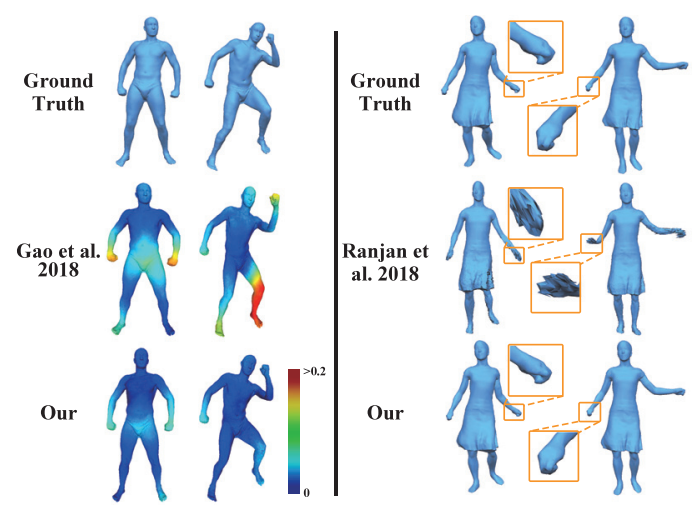

Figure 4. Qualitative comparison of reconstruction results for unseen data with [9] (left) and [26] (right). Reconstruction errors are color-coded on the left and the results on the right also show closeup views for more details. It can be seen that our method leads to more accurate reconstructions and the method of [26] suffers from easily noticeable artifacts.

parameters than the original MeshVAE. For Fat dataset with 6890 vertices for each shape, the original MeshVAE needs $129,745,920$ parameters, while ours needs 7, 941, 042 .

\subsection{Generation of Novel Models}

Once our network is trained, we can use the latent space and decoder to generate new shapes. We use the standard normal distribution $z \sim N(0, I)$ as the input to the trained decoder. It can be seen from Fig. 5 that our network is capable of generating reasonable new shapes. To prove that the generated shapes do not exist in the model dataset, we find the nearest shapes based on the average per-vertex Euclidean distance in the original datasets for visual comparison. It can be seen that the generated shapes are indeed new and different from any existing shape in the datasets. To show our conditional random generation ability, we train the network on the DYNA dataset from [23]. We use BMI+gender and motion as the condition to train the network. As shown in Fig. 6, our method is able to randomly generate models that are conditioned on the body shape '50007' - a male model with BMI 39.0 and conditioned on the action with the label 'One Leg Jump' including lifting a leg.

\subsection{Mesh Interpolation}

Our method can also be used for shape interpolation. This is also a way to generate new shapes. We linearly interpolate between the latent vectors of two shapes and the probabilistic decoder outputs a 3D deformation sequence. We compare our method on the SCAPE dataset [1] with a state-of-the-art data-driven deformation method [7], as shown in Fig. 7. We can see that the results by the datadriven method of [7] tend to follow the movement sequences from the original dataset which has similar start and end states, leading to redundant motions such as the swing of right arm. In contrast, our interpolation results give more reasonable motion sequences. We show more interpolation results in Fig. 9, including sequences between newly generated models and models beyond human bodies.

We compare our network with MeshVAE [31] to show the ability of our network for processing denser meshes. A comparison example for interpolation is shown in Fig. 8.

\section{Conclusions}

In this paper we introduced a newly defined pooling operation based on a modified mesh simplification algorithm and integrated it into a mesh variational auto-encoder ar- 


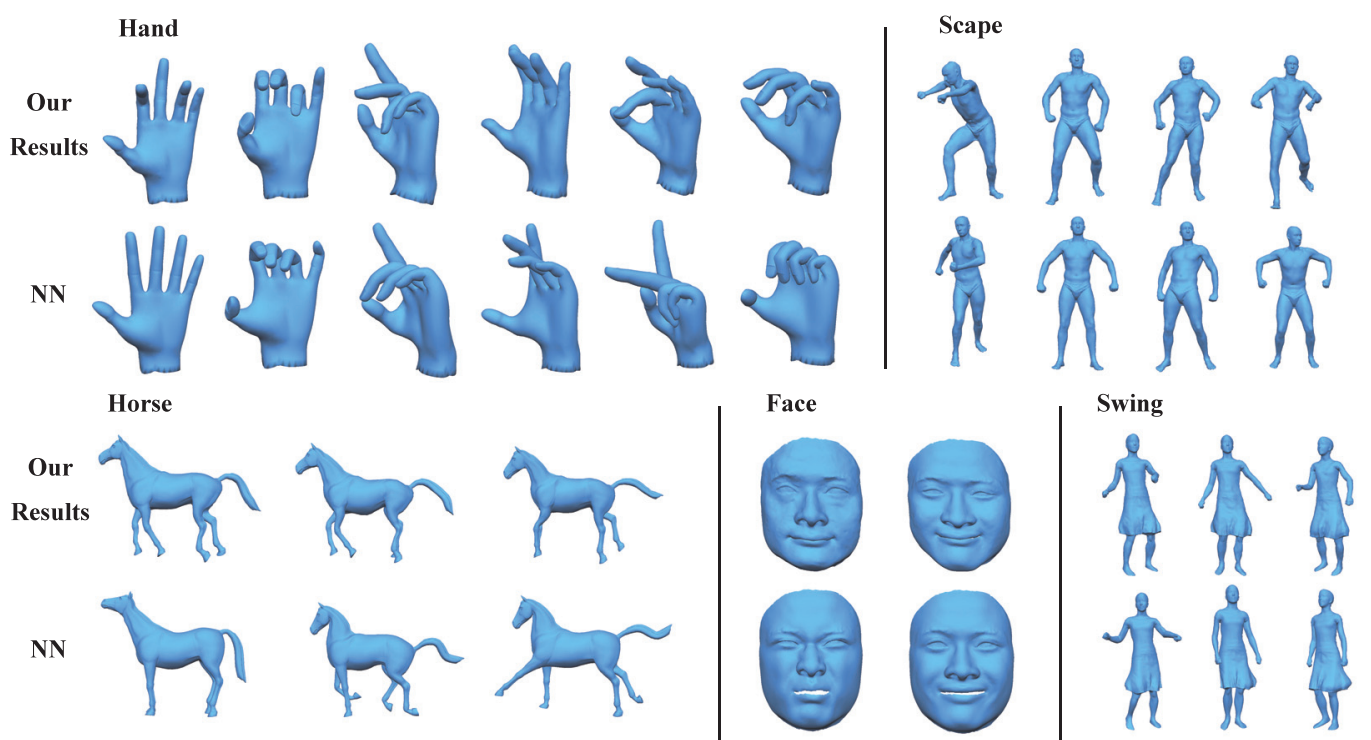

Figure 5. Randomly generated new shapes using our framework, along with their nearest neighbors (NN) in the original datasets.

Conditioned on Motion Sequence - One Leg Jump
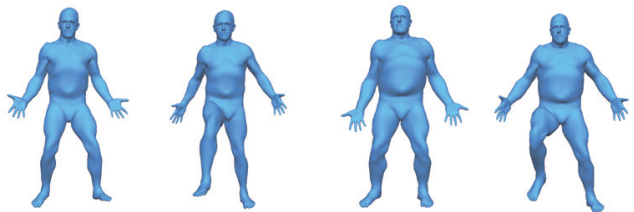

Conditioned on BodyShape - Male Model with BMI 39.0
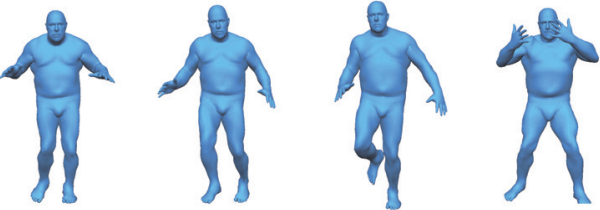

Figure 6. Conditional random generation of new shapes using our framework.

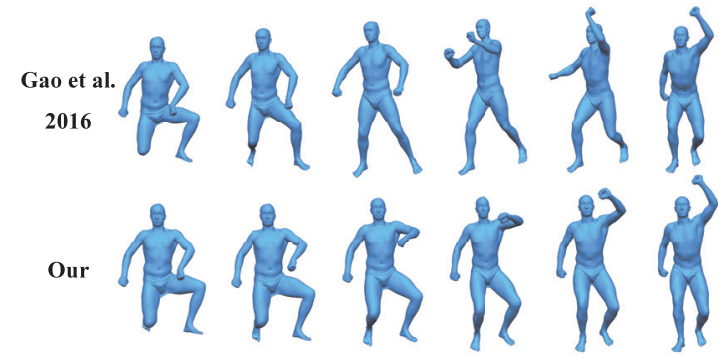

Figure 7. Comparison of mesh interpolation results with [7] (1st row). The models in the leftmost and rightmost columns are the input models to be interpolated.

chitecture, which uses per-vertex feature representations as inputs, and utilizes graph convolutions. Through extensive experiments we demonstrated that our generative model has better generalization ability. Compared to the original MeshVAE, our method can generate high quality deformable models with richer details. Our experiments also show that our method outperforms the state-of-the-art meth-

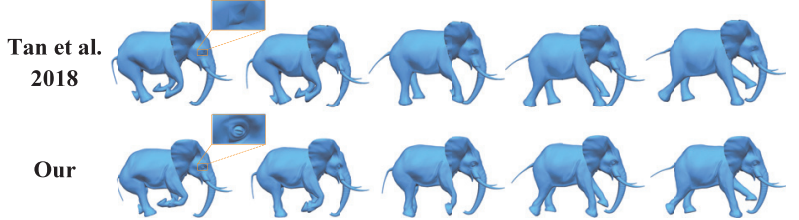

Figure 8. Interpolation comparison between MeshVAE [31] and our method. The original elephant model [29] has 42,321 vertices, which cannot be handled by MeshVAE due to memory restriction and therefore a simplified mesh with 5,394 vertices is used instead. Our method operates on the original mesh model and produces results with more details.

a)

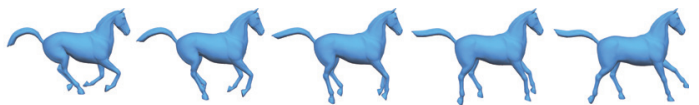

b)
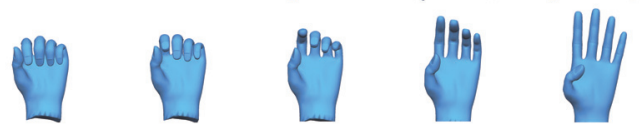

c)
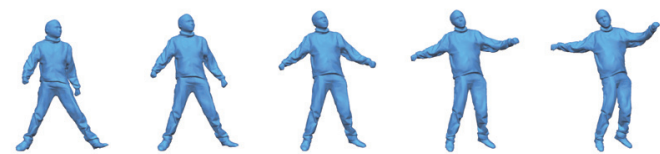

Figure 9. More interpolation results. (a)(b) more diverse shapes other than human bodies. (c) results interpolated between newly generated shapes.

ods in various applications including shape generation and shape interpolation. One of the limitations of our method is that it can only process homogeneous meshes. As future work, it is desirable to develop a framework capable of handling shapes with different topology as input. As our pooling is based on mesh simplification, it is not suitable for the cases that mesh simplification fails to generate reasonable outputs, such as non-watertight meshes and highly irregular mesh input. Further research on mesh simplification is required to deal with such cases. 


\section{Acknowledgement}

This work was supported by Beijing Municipal Natural Science Foundation (No. L182016), National Natural Science Foundation of China (No. 61872440 and No. 61828204), Newton Advanced Fellowship of Royal Society (No. NAF $\backslash$ R2 $\backslash 192151$ ), SenseTime Research Fund and Open Project Program of the National Laboratory of Pattern Recognition (NLPR).

\section{References}

[1] Dragomir Anguelov, Praveen Srinivasan, Daphne Koller, Sebastian Thrun, Jim Rodgers, and James Davis. SCAPE: shape completion and animation of people. ACM Transactions on Graphics (TOG), 24(3):408-416, 2005. 4, 5

[2] Davide Boscaini, Jonathan Masci, Emanuele Rodolà, and Michael Bronstein. Learning shape correspondence with anisotropic convolutional neural networks. In NIPS, pages 3189-3197, 2016. 2

[3] Davide Boscaini, Jonathan Masci, Emanuele Rodolà, Michael M Bronstein, and Daniel Cremers. Anisotropic diffusion descriptors. Computer Graphics Forum, 35(2):431441, 2016. 2

[4] Mario Botsch and Leif Kobbelt. A remeshing approach to multiresolution modeling. In SGP, pages 185-192, 2004. 2, 4,5

[5] Michael M. Bronstein, Joan Bruna, Yann LeCun, Arthur Szlam, and Pierre Vandergheynst. Geometric deep learning: going beyond Euclidean data. IEEE Signal Processing Magazine, 34(4):18-42, 2017. 2

[6] Michaël Defferrard, Xavier Bresson, and Pierre Vandergheynst. Convolutional neural networks on graphs with fast localized spectral filtering. In Advances in NIPS, pages 3844-3852, 2016. 2, 3

[7] Lin Gao, Shu-Yu Chen, Yu-Kun Lai, and Shihong Xia. Datadriven shape interpolation and morphing editing. Computer Graphics Forum, 36(8):19-31, 2017. 1, 5, 6

[8] Lin Gao, Yu-Kun Lai, Jie Yang, Ling-Xiao Zhang, Leif Kobbelt, and Shihong Xia. Sparse data driven mesh deformation. IEEE Transactions on Visualization and Computer Graphics, 2019. 1, 2, 4

[9] Lin Gao, Jie Yang, Yi-Ling Qiao, Yu-Kun Lai, Paul Rosin, Weiwei $\mathrm{Xu}$, and Shihong Xia. Automatic unpaired shape deformation transfer. ACM Transactions on Graphics (TOG), 37(6):1-15, 2018. 1, 2, 4, 5

[10] Lin Gao, Jie Yang, Tong Wu, Yu-Jie Yuan, Hongbo Fu, YuKun Lai, and Hao Zhang. SDM-NET: Deep generative network for structured deformable mesh. ACM Transactions on Graphics (TOG), 38(6), Nov. 2019. 2

[11] Michael Garland and Paul S. Heckbert. Surface simplification using quadric error metrics. In ACM SIGGRAPH, pages 209-216, 1997. 1, 2, 3, 4, 5

[12] Rana Hanocka, Amir Hertz, Noa Fish, Raja Giryes, Shachar Fleishman, and Daniel Cohen-Or. MeshCNN: A network with an edge. ACM Transactions on Graphics (TOG), 38(4):90:1-90:12, 2019. 2, 4, 5
[13] Mikael Henaff, Joan Bruna, and Yann LeCun. Deep convolutional networks on graph-structured data. arXiv preprint arXiv:1506.05163, 2015. 2

[14] Jingwei Huang, Haotian Zhang, Li Yi, Thomas Funkhouser, Matthias Niessner, and Leonidas J. Guibas. TextureNet: Consistent local parametrizations for learning from highresolution signals on meshes. In The IEEE Conference on Computer Vision and Pattern Recognition (CVPR), June 2019. 2

[15] Diederik P. Kingma and Jimmy Ba. Adam: A method for stochastic optimization. arXiv preprint arXiv:1412.6980, 2014. 4

[16] Diederik P. Kingma and Max Welling. Auto-encoding variational Bayes. arXiv preprint arXiv:1312.6114, 2013. 1

[17] Solomon Kullback and Richard A. Leibler. On information and sufficiency. The annals of mathematical statistics, 22(1):79-86, 1951. 4

[18] Or Litany, Alex Bronstein, Michael Bronstein, and Ameesh Makadia. Deformable shape completion with graph convolutional autoencoders. In The IEEE Conference on Computer Vision and Pattern Recognition (CVPR), June 2018. 1

[19] Haggai Maron, Meirav Galun, Noam Aigerman, Miri Trope, Nadav Dym, Ersin Yumer, Vladimir G Kim, and Yaron Lipman. Convolutional neural networks on surfaces via seamless toric covers. ACM Transactions on Graphics (TOG), 36(4):71, 2017. 2

[20] Jonathan Masci, Davide Boscaini, Michael Bronstein, and Pierre Vandergheynst. Geodesic convolutional neural networks on Riemannian manifolds. In ICCV workshops, pages 37-45, 2015. 2

[21] D. Maturana and S. Scherer. VoxNet: A 3D Convolutional Neural Network for Real-Time Object Recognition. In IROS, 2015. 1

[22] Thomas Neumann, Kiran Varanasi, Stephan Wenger, Markus Wacker, Marcus Magnor, and Christian Theobalt. Sparse localized deformation components. ACM Transactions on Graphics (TOG), 32(6):179, 2013. 4

[23] Gerard Pons-Moll, Javier Romero, Naureen Mahmood, and Michael J. Black. Dyna: A model of dynamic human shape in motion. ACM Transactions on Graphics (TOG), 34(4):120, 2015. 4, 5

[24] Charles R Qi, Hao Su, Kaichun Mo, and Leonidas J. Guibas. PointNet: Deep learning on point sets for 3D classification and segmentation. In The IEEE Conference on Computer Vision and Pattern Recognition (CVPR), 2017. 1

[25] Charles Ruizhongtai Qi, Li Yi, Hao Su, and Leonidas J Guibas. PointNet++: Deep hierarchical feature learning on point sets in a metric space. In Advances in NIPS, pages 5099-5108. Curran Associates, Inc., 2017. 2

[26] Anurag Ranjan, Timo Bolkart, Soubhik Sanyal, and Michael J. Black. Generating 3D faces using convolutional mesh autoencoders. In European Conference on Computer Vision (ECCV), pages 725-741. Springer International Publishing, 2018. 1, 2, 4, 5

[27] Yiru Shen, Chen Feng, Yaoqing Yang, and Dong Tian. Mining point cloud local structures by kernel correlation and 
graph pooling. In The IEEE Conference on Computer Vision and Pattern Recognition (CVPR), volume 4, 2018. 4, 5

[28] Kihyuk Sohn, Honglak Lee, and Xinchen Yan. Learning structured output representation using deep conditional generative models. In Advances in NIPS, pages 3483-3491, 2015. 4

[29] Robert W. Sumner and Jovan Popović. Deformation transfer for triangle meshes. ACM Transactions on Graphics (TOG), 23(3):399-405, 2004. 4, 6

[30] Qingyang Tan, Lin Gao, Yu-Kun Lai, Jie Yang, and Shihong Xia. Mesh-based autoencoders for localized deformation component analysis. In AAAI, 2018. 1, 2

[31] Qingyang Tan, Lin Gao, Yu-Kun Lai, and Shihong Xia. Variational autoencoders for deforming 3D mesh models. In The IEEE Conference on Computer Vision and Pattern Recognition (CVPR), June 2018. 1, 2, 4, 5, 6

[32] Daniel Vlasic, Ilya Baran, Wojciech Matusik, and Jovan Popović. Articulated mesh animation from multi-view silhouettes. ACM Transactions on Graphics (TOG), 27(3):97, 2008. 4

[33] Peng-Shuai Wang, Yang Liu, Yu-Xiao Guo, Chun-Yu Sun, and Xin Tong. O-CNN: Octree-based Convolutional Neural Networks for 3D Shape Analysis. ACM Transactions on Graphics (TOG), 36(4), 2017. 2

[34] Peng-Shuai Wang, Yang Liu, Yu-Xiao Guo, Chun-Yu Sun, and Xin Tong. Adaptive O-CNN: A Patch-based Deep Representation of 3D Shapes. ACM Transactions on Graphics (TOG), 37(6), 2018. 2

[35] Jiajun Wu, Chengkai Zhang, Tianfan Xue, William T Freeman, and Joshua B Tenenbaum. Learning a Probabilistic Latent Space of Object Shapes via 3D Generative-Adversarial Modeling. In Advances in NIPS, pages 82-90, 2016. 1

[36] Li Yi, Hao Su, Xingwen Guo, and Leonidas J Guibas. SyncSpecCNN: Synchronized spectral CNN for 3D shape segmentation. In The IEEE Conference on Computer Vision and Pattern Recognition (CVPR), pages 2282-2290, 2017. 2

[37] Jun-Yan Zhu, Taesung Park, Phillip Isola, and Alexei A. Efros. Unpaired image-to-image translation using cycleconsistent adversarial networks. In ICCV, 2017. 2 